\title{
Ajuste del Equilibrio Químico del Agua Potable con Tendencia Corrosiva por Dióxido de Carbono
}

\author{
Eduardo Trujillo, Verónica Martínez y Nadia S. Flores \\ Universidad Autónoma del Estado de México, Facultad de Ingeniería, Cerro de Coatepec s/n, \\ Ciudad Universitaria, 50130 Toluca, Edo. de México-México \\ (e-mail: etf@uaemex.mx, vmm@uaemex.mx)
}

\begin{abstract}
Resumen
En este trabajo se analiza el agua potable del principal tanque de distribución de la Ciudad de Toluca, Estado de México, para definir su tendencia corrosiva o incrustante y establecer condiciones de equilibrio químico de bajo costo. Se implementaron el modelo de Mojmir-Mach y los índices de Langelier y Ryznar en el software Agrlnc_Agua 2.0, para aplicarlo a datos fisicoquímicos de muestras de agua obtenidas durante seis meses. El agua cumple con las normas mexicanas para su uso y consumo humano. Sin embargo, mediante la identificación de productos de corrosión por difracción de rayos $\mathrm{X}$ y su composición elemental por microanálisis semicuantitativo, se comprobó que la presencia de $\mathrm{CO}_{2}$ tiende a corroer lentamente la infraestructura hidráulica. Se establecieron condiciones de equilibrio químico con base en hidróxido de calcio, carbonato de calcio y aireación. Todas estas variables pueden contribuir a proteger la infraestructura hidráulica y reducir la frecuencia de los mantenimientos, reemplazos y productos de corrosión.
\end{abstract}

Palabras clave: equilibrio químico, corrosión, incrustación, agua potable, método de Mojmir-Mach

\section{Chemical Equilibrium Adjustment of Drinking Water with Corrosive Tendency by Carbon Dioxide}

\begin{abstract}
On this research drinkable water from the main supply tank of the city of Toluca, México State, is analyzed in order to define its corrosive or scale forming tendency and set up low price chemical equilibrium conditions. The Mojmir Mach model and Langelier-Ryznar indexes were implemented in a software called Agrlnc_Agua 2.0 to apply them on physical-chemical data of water samples obtained during six months. This water fulfills the mexican standards for human use and consumption. However, using $X$ ray diffraction to identify corrosion products and using the elementary composition by semi quantitative micro analysis, it was proved that the presence of $\mathrm{CO}_{2}$ tends to corrode the hydraulic infrastructure in a slow way. Chemical equilibrium conditions were established using calcium hydroxide, calcium carbonate and aeration. All these variables can help in protecting the hydraulic infrastructure and to reduce the maintenance, replacement and corrosion products frequency.
\end{abstract}

Keywords: chemical equilibrium, corrosion, scale forming, drinkable water, Mojmir Mach method 


\section{INTRODUCCIÓN}

La infraestructura hidráulica de extracción y distribución de agua para uso y consumo humano suele sufrir importantes problemas de incrustación o corrosión interna y/o externa. La corrosión interna provoca el deterioro de la calidad del agua debido a los productos y contaminantes que se generan, disminuye el desempeño hidráulico y aumenta los costos de distribución y de mantenimiento de la red; en casos extremos, parte de la infraestructura queda inhabilitada temporalmente y por ende, se suspende el suministro.

Los fenómenos de corrosión y de incrustación, están relacionados directamente con las características fisicoquímicas del agua, su velocidad y tiempo de retención hidráulico, así como del material con el que el agua está en contacto (GQ, 2006a; Imran et al., 2005). Específicamente, la alcalinidad, $\mathrm{pH}$ y los residuos de desinfectantes son los principales factores que influyen en el proceso de corrosión (Rahman et al., 2007; NRC, 2006). Concentraciones bajas de alcalinidad favorecen la solubilidad del $\mathrm{CO}_{2}$, incrementando la agresividad de las aguas.

De acuerdo con Delion et al. (2004) y Rodier (1998), la corrosión o incrustación por agua está asociada a la concentración de $\mathrm{CO}_{2}$ libre, el cual influye en el comportamiento del carbonato de calcio $\left(\mathrm{CaCO}_{3}\right)$. Un agua en equilibrio químico mantiene la concentración adecuada de $\mathrm{CO}_{2}$ de tal forma que no se remueve la película de $\mathrm{CaCO}_{3}$ que protege a los materiales de la corrosión, ni se precipita $\mathrm{CaCO}_{3}$.

Aunque existen materiales que pueden sustituir a los propensos a la corrosión, suelen ser de menor resistencia mecánica e impactan la eficiencia del sistema; también existen aceros inoxidables o con contenidos de cobre o níquel que suelen resistir más tiempo a los efectos corrosivos, no obstante, su costo es elevado. En general, los materiales más utilizados en la infraestructura hidráulica son cobre, plomo, acero, hierro, cemento, asbesto, concreto y PVC.

Las aguas con alto contenido de $\mathrm{CO}_{2}$ corroen en frío las superficies metálicas pero también atacan al concreto o cemento-mortero; este tipo de material tiene asociados contaminantes como fibras de amianto (silicato de aluminio, alúmina y hierro) (GQ, 2006b; Schock, 1990).

En los sistemas de distribución cuya base es el hierro, se presentan componentes asociados a los productos de corrosión como geotita $(\alpha-\mathrm{FeOOH})$, lepidocrocita $(\gamma-\mathrm{FeOOH})$, magnetita $\left(\mathrm{Fe}_{3} \mathrm{O}_{4}\right)$, maghemita $\left(\alpha-\mathrm{Fe}_{2} \mathrm{O}_{3}\right)$, óxido ferroso $(\mathrm{FeO})$, siderita $\left(\mathrm{FeCO}_{3}\right)$, hidróxido ferroso $\left(\mathrm{Fe}(\mathrm{OH})_{2}\right)$, ferrihidrita $\left(5 \mathrm{Fe}_{2} \mathrm{O}_{3} .9 \mathrm{H}_{2} \mathrm{O}\right)$, carbonato de calcio $\left(\mathrm{CaCO}_{3}\right)$ (Zhang et al., 2008; Huang y Zhang, 2005; Sarin et al., 2004) y compuestos ferroso-férricos que incluyen $\mathrm{Cl}^{-}, \mathrm{SO}_{4}{ }^{2-} \circ \mathrm{CO}_{3}{ }^{2-}$; el tipo de mineral que se forma está determinado por la tasa de oxidación, el $\mathrm{pH}$, la concentración de $\mathrm{Fe}^{2+}$ así como de otras especies en solución (Sarin et al.,2004). Los contaminantes asociados al fierro o acero galvanizado o fundido son hierro, zinc, cadmio y plomo (GQ, 2006b; Schock, 1990).

Una vez que se ha determinado la presencia de aguas agresivas o incrustantes, su control requiere la elección de materiales adecuados, un buen programa de monitoreo y mantenimiento, y la modificación de las características químicas del agua. Cuando se trata de adecuar la calidad del agua, el ajuste del pH y la alcalinidad representan los métodos más comunes (FPTCDW, 2007), simples y económicos para controlar la corrosión, destacando la eliminación de $\mathrm{CO}_{2}$ por aereación, o el uso de $\mathrm{NaOH}, \mathrm{Ca}(\mathrm{OH})_{2}, \mathrm{Na}_{2} \mathrm{CO}_{3}$ o $\mathrm{NaHCO}_{3}(\mathrm{GQ}, 2006 \mathrm{a})$.

La Motta y Chinthankuntla (1996), diseñaron una torre de aeración para la estabilización química y control de la corrosión provocada por agua de consumo. Por otro lado, Letterman et al. (1991), indican que las capas de piedra caliza pueden mitigar la corrosión en pequeños sistemas, aunque la combinación de calicita con aire atmosférico puede generar mejores resultados.

La adición al agua de un álcali como el hidróxido de calcio (cal hidratada) o de carbonato de calcio, la equilibra de manera inocua, estabilizando el $\mathrm{pH}$ ácido causante de la corrosión; desde el punto de vista económico, este método de solución es muy bajo comparado con los costos generados por la rehabilitación de las redes de distribución, además es de fácil aplicación. 
Para poder establecer los programas de control y los tratamientos adecuados para abatir la corrosión o incrustación del agua, es importante determinar no solo cualitativa, sino también cuantitativamente su tendencia agresiva.

En México, para reducir las posibilidades de corrosión en la infraestructura hidráulica, la Comisión Nacional del Agua (CNA, 1994a, 1994b) recomienda que los materiales mantengan una película de $\mathrm{CaCO}_{3}$, asimismo, para evaluar la tendencia agresiva del agua, considera el uso de los siguientes índices: de saturación de Langelier, de agresividad, de estabilidad de Ryznar, de corrosión de Riddick, y de fuerza de conducción de Mc Cauley. De entre ellos, los índices de Langelier y Ryznar son los más utilizados. Estos modelos son considerados como cualitativos, ya que solo indican el carácter agresivo o incrustante del agua, sin determinar cuánto.

Otros índices utilizados para la caracterización del agua, incluyen la relación de Larson y la de Leroy (Delion et al. 2004), así como una relación modificada de Larson (Imran et al., 2005) que incorpora la electroneutralidad, temperatura, alcalinidad, y el tiempo de retención hidráulico.

Existen métodos que permiten determinar de manera cuantitativa la agresividad del agua, tal es el caso de Mojmir Mach (Allende, 1976; Trujillo et al., 2006; Alfaro et al., 2004) y Legrand-Poirier (Hamrouni y Dhabi, 2002), los cuales al tener un mayor sustento teórico proveen mejores resultados que los modelos cualitativos; sin embargo, debido a su complejidad y difícil aplicación, dichos modelos suelen ser poco utilizados.

En este trabajo, los dos índices más utilizados en México (Langelier y Ryznar) conjuntamente con el método de Mojmir Mach, se implementaron en una herramienta informática para determinar cualitativa y cualitativamente la tendencia corrosiva o incrustante del agua potable de un tanque de distribución, y proponer una alternativa de bajo costo para la adecuación de su calidad química, de tal forma que pueda proteger la infraestructura hidráulica, sin alterar la condición de agua para uso y consumo humano.

\section{MATERIALES Y MÉTODOS}

Se eligió el principal tanque de distribución que abastece agua potable a la Ciudad de Toluca, Estado de México, debido a las manifestaciones corrosivas detectadas en la infraestructura hidráulica de distribución. El tanque recibe un flujo de agua superficial potabilizada, así como el aporte de agua de siete pozos que se homogenizan en dicho tanque. Para aplicar los modelos de equilibrio químico del agua, se realizaron muestreos mensuales durante medio año, abarcando épocas de estiaje y lluvia.

\section{Mediciones y análisis de laboratorio}

Se llevaron a cabo seis muestreos. Se realizaron mediciones in situ de temperatura, $\mathrm{pH}$, conductividad eleéctrica, y cloro residual, asimismo, se determinaron en laboratorio los parámetros transitorios (acidez, alcalinidad, dureza, DQO, DBO), las especies aniónicas (N-nitratos, N-nitritos, Namoniacal, cloruros, sulfatos, fosfatos), catiónicas (calcio, magnesio, sodio, potasio), sólidos (disueltos totales, disueltos fijos, disueltos volátiles, suspendidos totales, suspendidos fijos, suspendidos volátiles) y parámetros microbiológicos (coliformes totales y fecales). Las mediciones y determinaciones, se realizaron con base en las técnicas de análisis establecidas en las Normas Oficiales Mexicanas vigentes y por la APHA-AWWA-WPFC (1998).

La medición de la temperatura, conductividad eléctrica y $\mathrm{pH}$, se realizó con un termómetro de mercurio y un potenciómetro. El cloro residual se midió por colorimetría durante todo el periodo de muestreo.

\section{Métodos cualitativos}

El índice de Langelier proporciona un valor cualitativo sobre la estabilidad del agua, aplicando la diferencia entre el pH medido del agua y el pH de equilibrio o de saturación (CNA, 1994a, 1994b): 
$\left(\mathrm{I}_{\mathrm{L}}=\mathrm{pH}-\mathrm{pH}_{\mathrm{s}}\right)$

Si el índice es igual con cero, el agua está en equilibrio químico, si es positivo es incrustante, y si es negativo es corrosiva. El índice de Langelier no proporciona una estimación del grado de corrosión o incrustación del agua en estudio (Allende, 1976).

El índice de estabilidad de Ryznar (CCA, 2005), toma como base los mismos parámetros que Langelier, pero siempre da un valor positivo, el cual provee una idea del nivel corrosivo o incrustante del agua.

$\left(I_{R}=2 \mathrm{pH}_{\mathrm{s}}-\mathrm{pH}\right)$

El carácter del agua se determina con base en los siguiente criterios: $4 \leq \mathrm{I}_{\mathrm{R}}<5$, fuertemente incrustante; $5 \leq I_{R}<6$, moderadamente incrustante; $6 \leq I_{R}<7$, poco incrustante o corrosiva; $7 \leq I_{R}<$ 7.5 , corrosiva; $7.5 \leq I_{R}<9$, fuertemente corrosiva; $9 \leq I_{R}$, muy corrosiva.

\section{Método cuantitativo}

El método de Mojmir Mach (Allende, 1976) se fundamenta en el índice de Langelier y en el gráfico de Tillmans, Mach incorpora la fuerza iónica $(\mu)$ al índice de Langelier, y considera la aportación de los denominados iones ajenos (cationes y aniones presentes en el agua y diferentes al calcio, bicarbonatos, carbonatos y protones). El pH de equilibrio o saturación $\left(\mathrm{pH}_{\mathrm{s}}\right)$ del agua se obtiene de:

$\mathrm{pH}_{\mathrm{s}}=0.6301+\mathrm{pK}_{2}-\mathrm{pK}_{\mathrm{ps}}-\log [\mu-\mathrm{A}]-\log [\mu-\mathrm{D}]+2.5 \varepsilon$

donde, $\mathrm{pK}_{2}$ es la segunda constante de disociación del ácido carbónico, $\mathrm{pK}_{\mathrm{ps}}$ el producto de solubilidad del carbonato de calcio, $\mathrm{A}=3\left[\mathrm{Cat}^{++}\right]+\left[\mathrm{Cat}^{+}\right]+\left[\mathrm{An}{ }^{\circ}\right], \mathrm{D}=4\left[\mathrm{An}^{\overline{ }}\right]-0.5\left[\mathrm{Cat}^{+}\right]+1.5\left[\mathrm{An}^{-}\right]$, $\mathrm{Cat}^{++}$son los cationes divalentes, $\mathrm{Cat}^{+}$los cationes monovalentes, $\mathrm{An}^{=}$los aniones divalentes, $\mathrm{An}^{-}$los

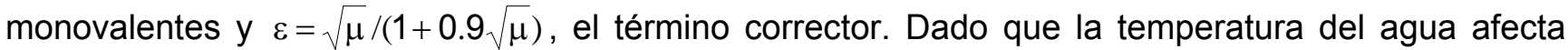
sus propiedades agresivas, las constantes de equilibrio pueden calcularse con base en las ecuaciones presentadas por Masschelein (1982).

El índice de saturación de Langelier calculado con el pH de saturación del modelo de Mojmir Mach, se interpreta de la misma forma que el índice original.

Considerando la fuerza iónica en equilibrio, es posible determinar las concentraciones de calcio y bicarbonatos con base en:

$\left[\mathrm{Ca}^{++}\right]=\frac{1}{3}(\mu-\mathrm{A})$

$\left[\mathrm{HCO}_{3}^{-}\right]=\frac{2}{3}(\mu-\mathrm{A})$

Por lo tanto, la tendencia del agua puede determinarse a través de la fuerza iónica y las concentraciones de calcio, o bicarbonatos:

$$
\begin{aligned}
& \operatorname{Agr}_{1}=\frac{1}{3}\left(\mu_{\text {eq }}-\mu_{\text {real }}\right) \\
& \operatorname{Agr}_{2}=\left[\mathrm{Ca}^{++}\right]_{\text {eq }}-\left[\mathrm{Ca}^{++}\right]_{\text {real }}
\end{aligned}
$$




$$
\mathrm{Agr}_{3}=\left[\mathrm{HCO}_{3}^{-}\right]_{\text {eq }}-\left[\mathrm{HCO}_{3}^{-}\right]_{\text {real }}
$$

Cuando el valor de Agr (ecuaciones 6, 7 y 8 ) es igual con cero el agua se encuentra en equilibrio, si es positivo el agua es considerada como corrosiva, si es negativo, el agua es considerada como incrustante.

La representación gráfica del equilibrio de saturación del método de Mojmir Mach tiene como base el diagrama de equilibrio de Tillmans, a través de un sistema de coordenadas con ordenada $\left[\mathrm{H}_{2} \mathrm{CO}_{3}\right]$ y abscisa $1 / 2\left[\mathrm{HCO}_{3}{ }^{-}\right]$. En este sistema de coordenadas se pueden representar las concentraciones determinadas de $\left[\mathrm{H}_{2} \mathrm{CO}_{3}\right]$ y $\left[\mathrm{HCO}_{3}^{-}\right]$obtenidas a partir de la acidez y alcalinidad del agua respectivamente, incluyendo los iones ajenos en el equilibrio de saturación. Si el punto está por debajo de la curva de equilibrio, se clasifica a ésta como un agua incrustante, y cuando está arriba de la curva, como agresiva. El bióxido de carbono total se puede obtener mediante:

$$
\left[\mathrm{CO}_{2}\right]_{\mathrm{t}}=\left[\mathrm{HCO}_{3}^{-}\right]+\left[\mathrm{H}_{2} \mathrm{CO}_{3}\right]
$$

Utilizando las concentraciones iniciales y de equilibrio de ácido carbónico y bicarbonatos, se determina la concentración que se requiere adicionar de hidróxido o carbonato de calcio por litro para obtener un agua en equilibrio químico.

\section{Software de apoyo}

El índice de saturación de Langelier, el índice de estabilidad de Ryznar, así como el método cuantitativo de Mojmir Mach se implementaron en una herramienta informática denominada Agrlnc_Agua 2.0 - Agresividad e Incrustabilidad del Agua. La codificación se realizó bajo ambiente Windows ${ }^{\circledR}$ en el lenguaje de programación Borland ${ }^{\circledR}$ Delphi ${ }^{\circledR}$ 2005. La interfaz gráfica de usuario (Fig. 1) se diseñó considerando aspectos de usabilidad de tal forma que se facilitara su uso.

Para determinar la naturaleza agresiva o incrustante del agua, se requieren los datos de concentraciones en $\mathrm{mg} / \mathrm{L}$ de los cationes, aniones, especies encontradas en el agua y sólidos disueltos totales, provenientes de los reportes de laboratorio, así como la temperatura del agua, y su $\mathrm{pH}$, estos datos se introducen directamente en la interfaz, o mediante un archivo tipo texto.

Agrlnc_Agua 2.0 indica de forma gráfica y numérica, el tipo de agua y determina el punto necesario para mantenerla en equilibrio químico. Los resultados numéricos pueden almacenarse en un archivo que es posible leer directamente en Microsft ${ }^{\circledR}$ Word ${ }^{\circledR}$, mientras que las gráficas se almacenan en archivos con formato de mapa de bits (Bit MaP, BMP), que también pueden incorporarse a Word ${ }^{\circ} \mathrm{O}$ manejarse en cualquier visualizador de imágenes.

\section{Caracterización del material adherido al sistema de distribución}

Derivado de las actividades de mantenimiento a la infraestructura del tanque, fue posible obtener muestras de los materiales adheridos en un tramo de tubería de acero (Fig. 1a) y concreto reforzado (Fig. 2a), que estuvieron en servicio por aproximadamente 20 años. La caracterización de las dos muestras se realizó para determinar su morfología y componentes.

Para obtener información cualitativa de los componentes de los sólidos de las muestras se aplicó difracción de rayos $X$ mediante un difractómetro acoplado a un tubo de rayos $X$ con ánodo de cobre. Mediante microscopia electrónica de barrido se determinó la morfología de los materiales utilizando un microscopio de alto vacío. El microanálisis elemental se realizó mediante una sonda acoplada al microsopio para determinar de manera semicuantitativa su composición. 


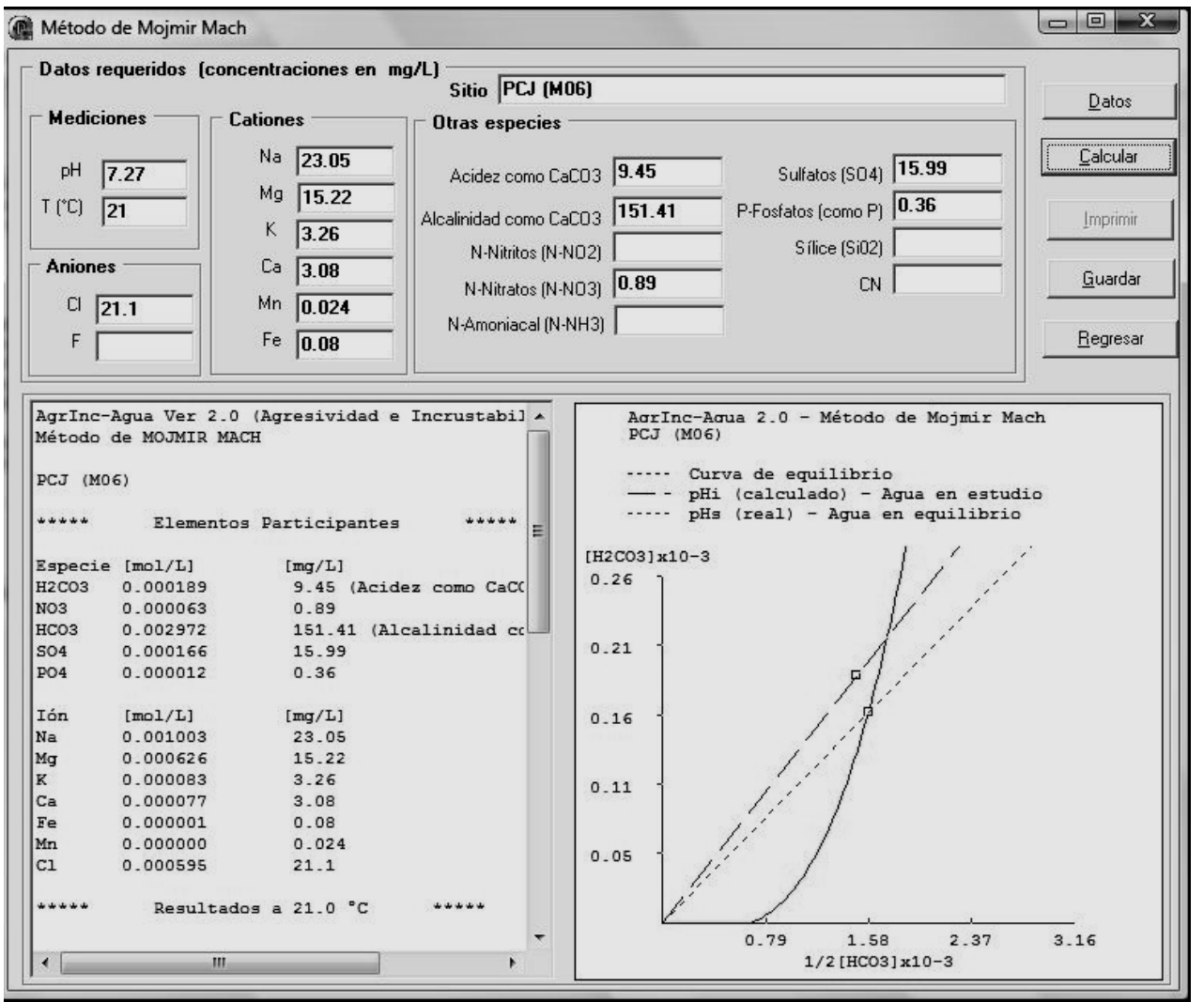

Fig. 1: Interfaz de usuario del método de Mojmir-Mach, en Agrlnc_Agua 2.0

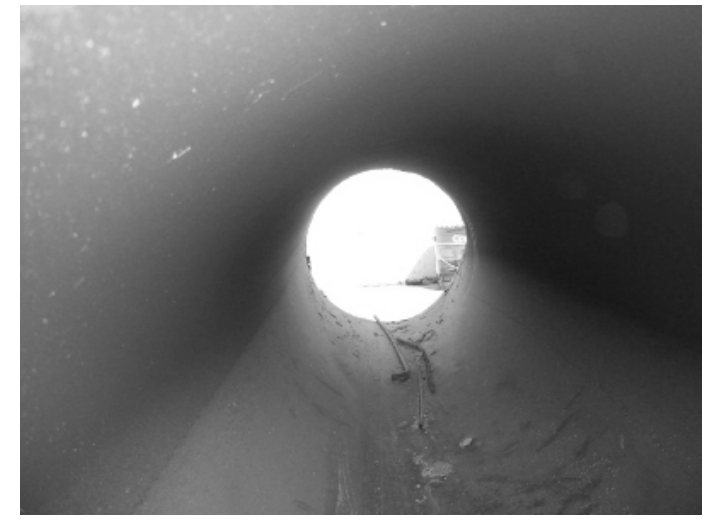

a) Concreto reforzado

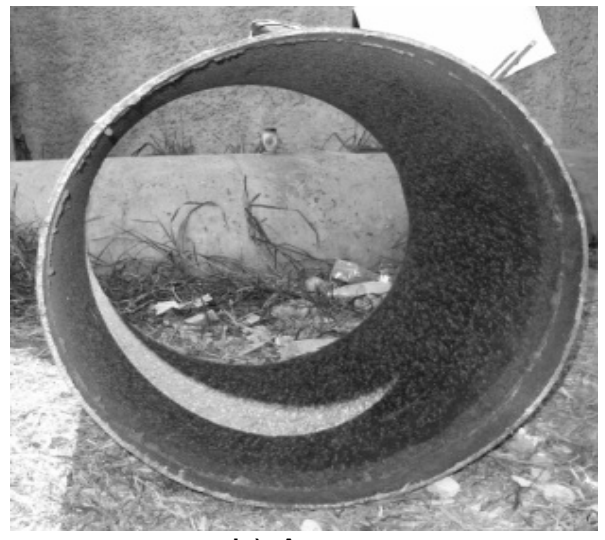

b) Acero

Fig. 2: Tramos de tubería de la infraestructura del tanque de distribución

\section{RESULTADOS Y DISCUSIÓN}

La tabla 1 muestra la composición fisicoquímica y microbiológica obtenida de las muestras de agua del tanque de distribución. Los coliformes totales y fecales corresponden a valores menores a 2 $\mathrm{NMP} / 100 \mathrm{~mL}$, no detectables con la técnica del número más probable. De acuerdo con la Norma Oficial Mexicana (NOM-127-SSA1-1994, 2000), el agua del tanque cumple los requerimientos para uso y consumo humano.

Los resultados correspondientes a la aplicación del software Agrlnc_Agua 2.0 se presentan en la tabla 2, donde se muestra la tendencia corrosiva o incrustante del agua, así como las condiciones de equilibrio químico mediante carbonato de calcio, e hidróxido de calcio.

De acuerdo con el índice de Ryznar, el agua se identifica como muy agresiva, los métodos de Langelier y Mojmir Mach confirman esa tendencia, además, los pH de saturación de los métodos cualitativos y cuantitativos resultan ser similares. 
El método de Mojmir Mach presentó los tres valores de agresividad positivos para las muestras de los primeros cinco meses, en el último mes, los valores de agresividad calculados con base en las concentraciones de calcio y la fuerza iónica resultan contradictorios de acuerdo con el valor obtenido con los bicarbonatos y con los resultados de los dos métodos cualitativos.

Los resultados discordantes pueden ser atribuidos a los incrementos observados en las concentraciones de calcio y ácido carbónico; en este caso, no es adecuado abatir la concentración de ácido carbónico a través de la agregación de un álcali.

Tabla 1: Parámetros fisicoquímicos y microbiológicos del agua del tanque en estudio.

\begin{tabular}{|c|c|c|c|c|c|c|c|}
\hline \multirow[b]{2}{*}{ Parámetro } & \multirow[b]{2}{*}{ Unidades } & \multicolumn{6}{|c|}{ Mes } \\
\hline & & 1 & 2 & 3 & 4 & 5 & 6 \\
\hline Hora & hh:mm & 10:34 & 09:38 & 10:15 & $11: 14$ & 10:00 & $10: 10$ \\
\hline Temperatura de la muestra & ${ }^{\circ} \mathrm{C}$ & 17.7 & 14.4 & 17.4 & 19 & 19 & 19 \\
\hline Conductividad eléctrica & $\mu \mathrm{S} / \mathrm{cm}$ & 207 & 239 & 236 & 260 & 230 & 170 \\
\hline $\mathrm{pH}$ & $\mathrm{pH}$ & 6.5 & 6.9 & 7.1 & 7.3 & 7.9 & 6.7 \\
\hline Cloro & $\mathrm{mg} / \mathrm{L}$ & 1.5 & 1 & 1.5 & 1.5 & 1 & 1 \\
\hline Alcalinidad & $\mathrm{mg} / \mathrm{L} \mathrm{CaCO}_{3}$ & 60.5 & 66.8 & 104.4 & 76.7 & 138.8 & 80.2 \\
\hline Acidez & $\mathrm{mg} / \mathrm{L} \mathrm{CaCO}_{3}$ & 5.5 & 4.3 & 7.2 & 5.4 & 6.8 & 19.2 \\
\hline Dureza & $\mathrm{mg} / \mathrm{L} \mathrm{CaCO}_{3}$ & 31.4 & 61.7 & 74.0 & 78.2 & 77.2 & 78.7 \\
\hline N-nitratos & $\mathrm{mg} / \mathrm{L} \mathrm{N}^{-\mathrm{NO}^{-}}{ }_{3}$ & 0.63 & 1.32 & 0.86 & 0.75 & 0.78 & 0.13 \\
\hline N-nitritos & $\mathrm{mg} / \mathrm{L} \mathrm{N}^{-\mathrm{NO}^{-}}{ }_{2}$ & 0.002 & 0.001 & 0.003 & $<0.001$ & $<0.001$ & 0.005 \\
\hline Cloruros & $\mathrm{mg} / \mathrm{L} \mathrm{Cl}^{-}$ & 12.9 & 9.0 & 8.2 & 5.7 & 5.1 & 7.1 \\
\hline Sulfatos & $\mathrm{mg} / \mathrm{L} \mathrm{SO}_{4}^{-2}$ & 16.5 & 15.2 & 16.1 & $\begin{array}{r}0.1 \\
17.4\end{array}$ & 15.6 & 16.1 \\
\hline Fosfatos & $\mathrm{mg} / \mathrm{L} \mathrm{P}_{-} \mathrm{PO}^{-3}{ }_{4}$ & 0.03 & 0.02 & 0.04 & 0.02 & 0.04 & 0.034 \\
\hline Calcio & $\mathrm{mg} / \mathrm{L} \mathrm{Ca}$ & 11.7 & 13.2 & 13.8 & 12.7 & 15.0 & 21.5 \\
\hline Magnesio & $\mathrm{mg} / \mathrm{L} \mathrm{Mg}$ & 7.6 & 8.7 & 9.0 & 8.2 & 10.1 & 10.6 \\
\hline Hierro & $\mathrm{mg} / \mathrm{L} \mathrm{Fe}$ & $<0.08$ & $<0.08$ & $<0.08$ & $<0.08$ & $<0.08$ & $<0.08$ \\
\hline Potasio & $\mathrm{mg} / \mathrm{L} \mathrm{K}$ & 3.4 & 3.6 & 3.7 & 3.7 & 3.7 & 3.4 \\
\hline Sodio & $\mathrm{mg} / \mathrm{L} \mathrm{Na}$ & 9.8 & 11.3 & 25.4 & 12.5 & 14.0 & 37.1 \\
\hline Sólidos disueltos totales & $\mathrm{mg} / \mathrm{L}$ SDT & 202 & 153 & 203 & 233 & 178 & 142 \\
\hline Sólidos suspendidos totales & $\mathrm{mg} / \mathrm{L}$ SST & 0 & 0 & 4 & 2 & 2 & 3 \\
\hline Coliformes totales & NMP/100 mL & $<2$ & $<2$ & $<2$ & $<2$ & $<2$ & $<2$ \\
\hline Coliformes fecales & $\mathrm{NMP} / 100 \mathrm{~mL}$ & $<2$ & $<2$ & $<2$ & $<2$ & $<2$ & $<2$ \\
\hline Dióxido de carbono & $\mathrm{mg} / \mathrm{L} \mathrm{CO} 2$ & 4.8 & 21.4 & 6.3 & 4.8 & 5.9 & 6.9 \\
\hline
\end{tabular}

Tabla 2: Resultados de la aplicación de Agrlnc_Agua 2.0.

\begin{tabular}{|c|c|c|c|c|c|c|c|}
\hline & \multicolumn{6}{|c|}{ Mes } \\
\hline & & 1 & 2 & 3 & 4 & 5 & 6 \\
\hline pH medido & $\mathrm{pH}$ & 6.5 & 6.9 & 7.0 & 7.3 & 7.9 & 6.7 \\
\hline \multirow{3}{*}{$\begin{array}{c}\text { Métodos } \\
\text { Cualitativos }\end{array}$} & $\mathrm{pHs}$ & 8.4 & 8.4 & 8.1 & 8.3 & 7.9 & 8 \\
\hline & I. Langelier & -2 & -1.5 & -1 & -1 & -0.02 & -1.3 \\
\hline & I. Ryznar & 10.4 & 9.9 & 9.2 & 9.2 & 7.9 & 9.3 \\
\hline \multirow{7}{*}{$\begin{array}{c}\text { Método } \\
\text { Cuantitativo } \\
\text { M. Mach } \\
\text { (mmoles/L) }\end{array}$} & $\mathrm{pHs}$ & 8.3 & 8.4 & 8.1 & 8.2 & 7.7 & 8.6 \\
\hline & I. Langelier & -0.8 & -0.8 & -0.6 & -0.7 & -0.1 & -1.6 \\
\hline & Agr1 & 0.17 & 0.08 & 0.12 & 0.13 & 0.35 & -0.14 \\
\hline & Agr2 & 0.20 & 0.08 & 0.12 & 0.15 & 0.50 & -0.39 \\
\hline & Agr3 & 0.09 & 0.07 & 0.10 & 0.08 & 0.04 & 0.37 \\
\hline & $\mathrm{H}_{2} \mathrm{CO}_{3}$ inicial & 0.11 & 0.09 & 0.14 & 0.11 & 0.14 & 0.38 \\
\hline & $\mathrm{H}_{2} \mathrm{CO}_{3 \mathrm{eq}}$ & 0.02 & 0.02 & 0.04 & 0.03 & 0.12 & 0.02 \\
\hline \multirow{2}{*}{$\begin{array}{c}\text { Equilibrio } \\
\text { (mg/L) }\end{array}$} & $\mathrm{Ca}(\mathrm{OH})_{2}$ & 3.37 & 2.62 & 3.72 & 3.06 & 0.51 & \\
\hline & $\mathrm{CaCO}_{3}$ & 9.1 & 7.08 & 10.06 & 8.26 & 1.37 & 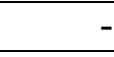 \\
\hline
\end{tabular}


Las muestras de los materiales adheridos a la superficie de la tubería de acero y concreto reforzado que forman parte de la infraestructura del tanque distribución, fueron identificadas como AFe y CFe respectivamente, y fueron analizadas para confirmar los efectos corrosivos del agua potable estudiada.

El difractograma obtenido de AFe (Fig 3a) revela la presencia de magnetita, lepidocrocita, geotita y óxido ferroso, estos componentes corresponde a la primera capa de los productos del proceso de corrosión (top surface layer) que se depositan a través de su precipitación y una oxidación lenta que puede generar $\mathrm{Fe}^{2+}$, y es originada por $\mathrm{CO}_{2}$ demostrando así el carácter agresivo del agua.

En función del pH del agua se puede favorecer la formación de geotita o magnetita (Sarin et al., 2004), como productos insolubles de la corrosión, lo cual coincide con los valores obtenidos en este trabajo ( $\mathrm{pH}$ de 6.5 a 7.9) y que se muestran en la tabla 1.

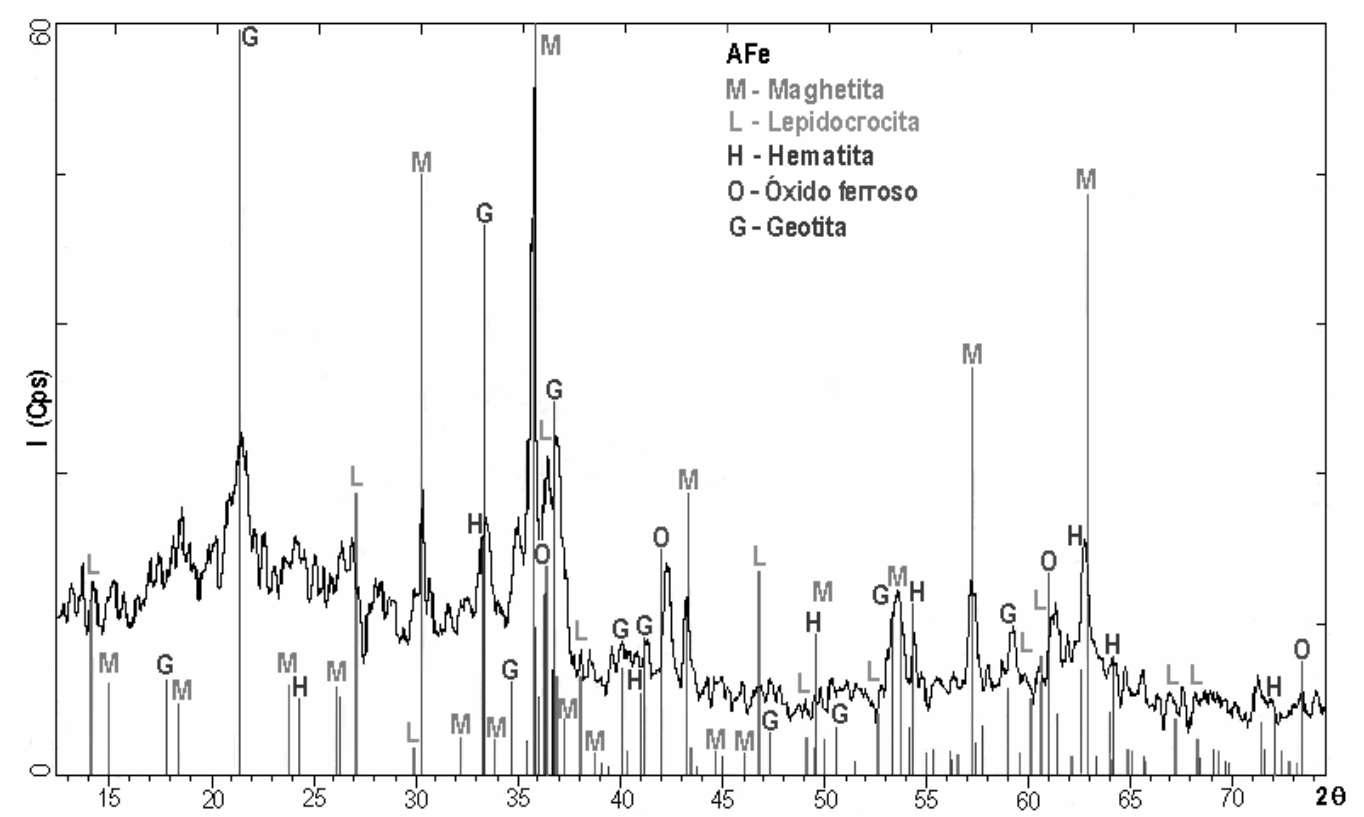

a) Muestra AFe

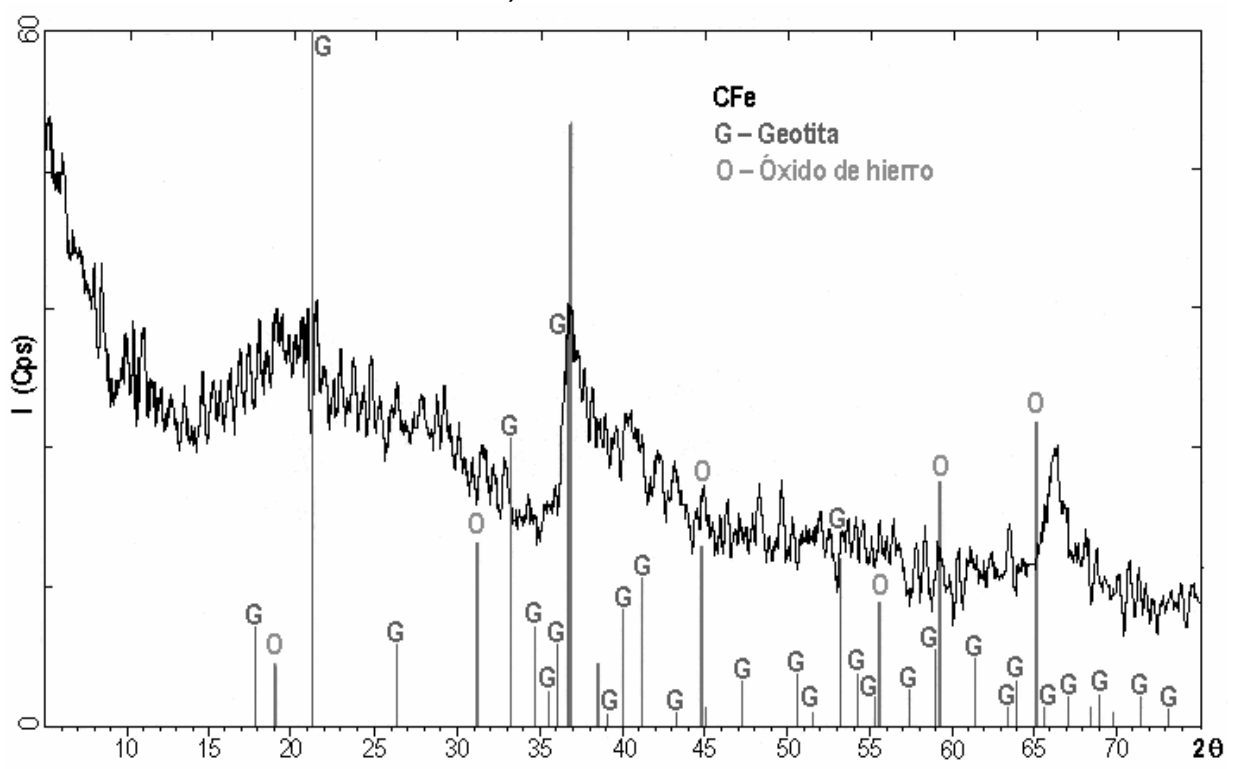

b) Muestra CFe

Fig. 3. Difractogramas de los productos de corrosión AFe y CFe 
El material incrustado en CFe es un polvo de color rojo intenso menos cristalino en donde se distinguen geotita y óxido de hierro (Fig $3 b$ ), los cuales corresponden a sólidos insolubles productos de corrosión que se precipitan para formar la capa conocida como concoidea (shell-like layer) (Sarin et al., 2004) y la capa de un sistema de corrosión provocado por $\mathrm{ClO}_{2}$ (dióxido de cloro) (Zhang et al., 2008).

Los resultados de la microscopia electrónica de barrido y microanálisis elemental en AFe (Fig. 4a y 5a) muestran la presencia de cristales irregulares cubiertos por una fase amorfa, muy similar a la fase principal que cubre a las partículas del material CFe (Fig. 4b y 5b). La muestra AFe está compuesta principalmente por oxígeno, hierro y silicio, en menor proporción por carbono, aluminio, fósforo, cloro y manganeso. En CFe hay presencia de aluminio, silicio, oxígeno y manganeso, en menor proporción se encontró calcio, hierro, magnesio, azufre, fósforo y titanio.

La presencia de elementos adicionales a los productos de oxidación del hierro corresponden a productos de precitación de silicatos $\left(\mathrm{SiO}_{4}{ }^{4-}\right)$, fosfatos $\left(\mathrm{PO}_{4}{ }^{3-}\right)$ y carbonatos $\left(\mathrm{CO}_{3}{ }^{2-}\right)$ debido a los valores de alcalinidad $\left(60.4\right.$ - $138.8 \mathrm{mg} / \mathrm{L}$ como $\left.\mathrm{CaCO}_{3}\right)$ del agua del tanque; la presencia de aluminio se debe a los residuos del proceso de coagulación del agua que llega al tanque (Trejo et al., 2004) y que se precipita (NRC, 2006); con respecto a CFe, el aluminio es un contaminante primario derivado del material $(\mathrm{GQ}, 2006 \mathrm{~b})$.

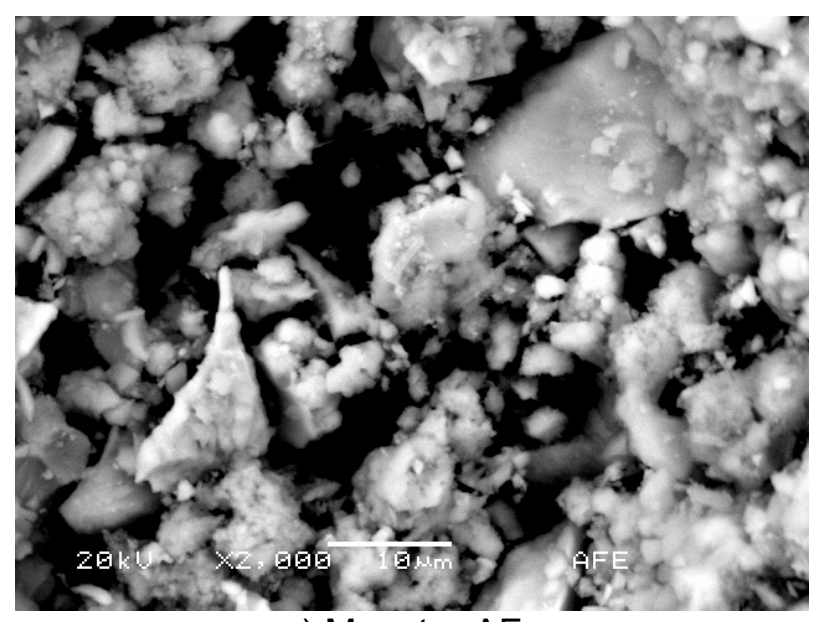

a) Muestra AFe

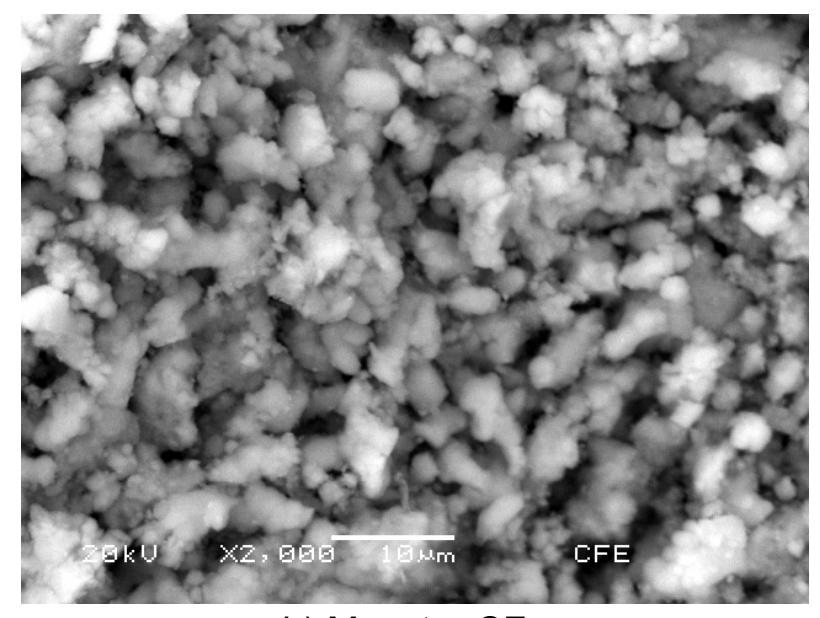

b) Muestra CFe

Fig. 4: Imágenes obtenidas por microscopia electrónica de barrido de los productos de corrosión AFe y CeF. 2000x.

El manganeso se encuentra en mayor proporción en CFe debido a que se libera en el proceso de corrosión en los materiales metálicos, ya que se encuentra combinado con el hierro. Los sólidos depositado en el concreto reforzado son polvos finos de color rojo intenso, en época de estiaje la interrupción del abastecimiento debe ser muy breve, de lo contrario, dichos sólidos podrían desprenderse dándole color al agua, además se podría incrementar el contenido de manganeso y eventualmente provocar daños a la salud derivado de su consumo.

Los resultados anteriores confirman la naturaleza agresiva del agua del tanque, que teóricamente fue determinada por los índices de Langelier y Ryznar, así como por el método de Mojmir-Mach, con base en este último, se proponen alternativas para equilibrar el contenido de $\mathrm{CO}_{2}$ en el agua del tanque mediante $\mathrm{Ca}(\mathrm{OH})_{2}$ y $\mathrm{CaCO}_{3}$.

Para asegurar que las propuestas para equilibrar el agua son adecuadas, se construyeron los diagramas de distribución de especies (Fig. 6), con el programa de cómputo Medusa (Puigdomench et al., 1999), para las condiciones iniciales y de equilibrio de fuerza iónica, concentración de bicarbonatos y calcio, para el primer y último mes. 


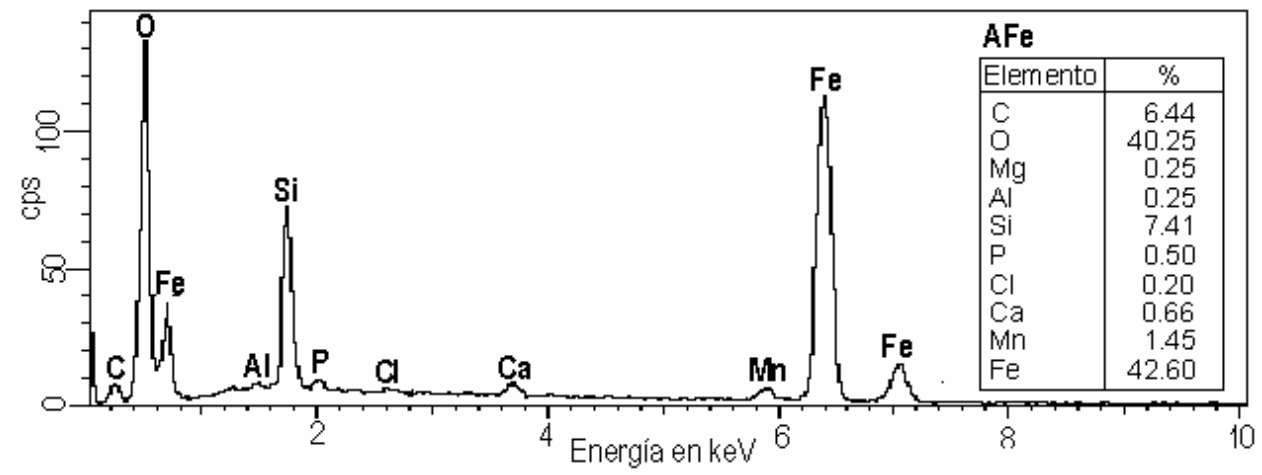

a) Muestra $\mathrm{AFe}$

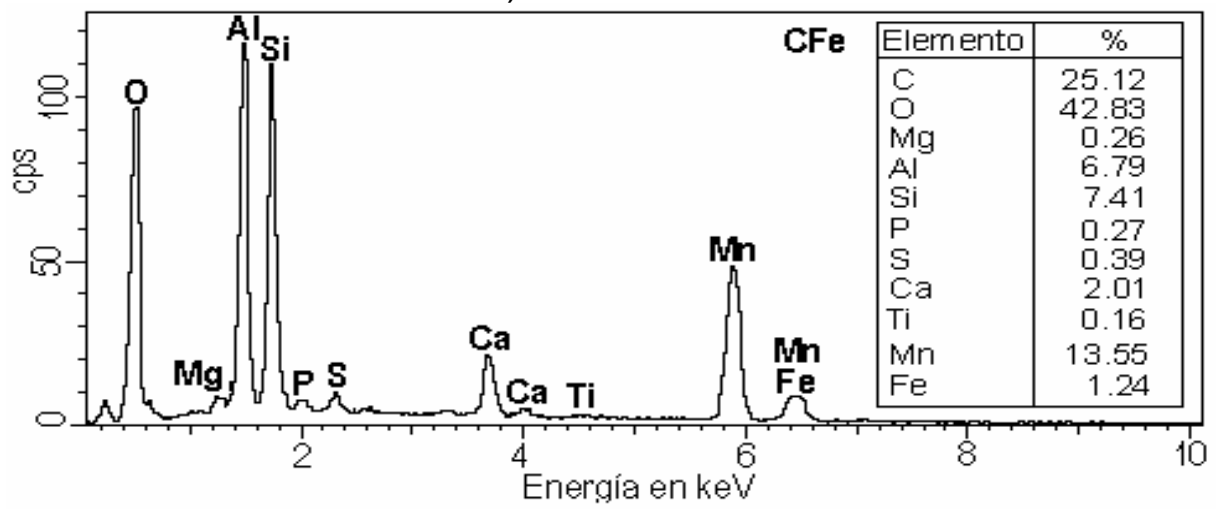

b) Muestra CFe

Fig. 5: Microanálisis elemental de los productos de corrosión AFe y CeF

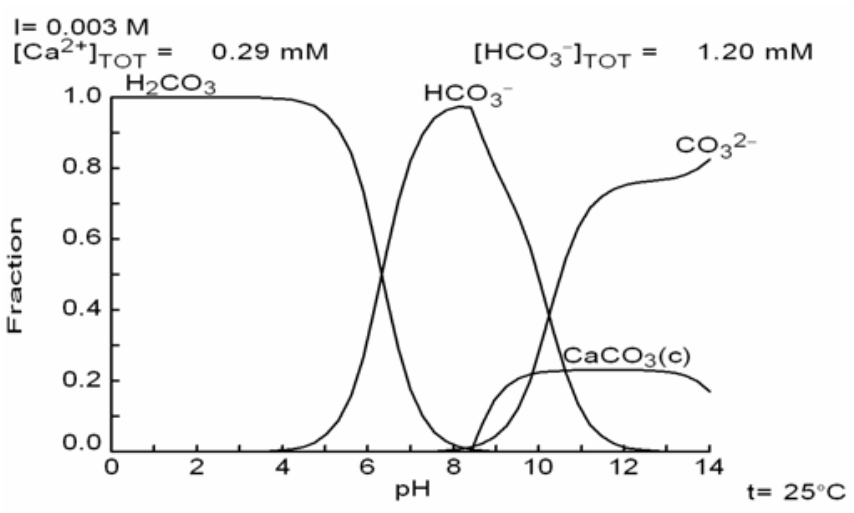

a) En condiciones iniciales del Mes 1

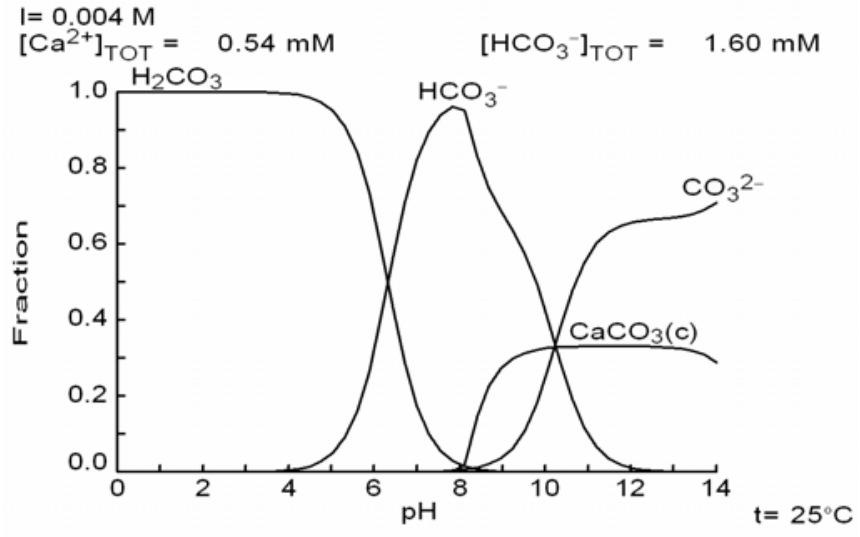

c) En condiciones iniciales del Mes 6

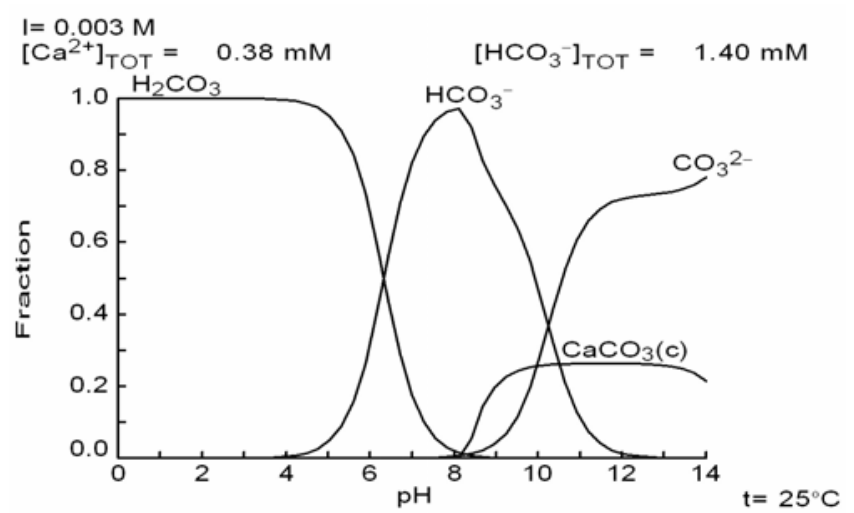

b) En condiciones de equilibrio del Mes 1

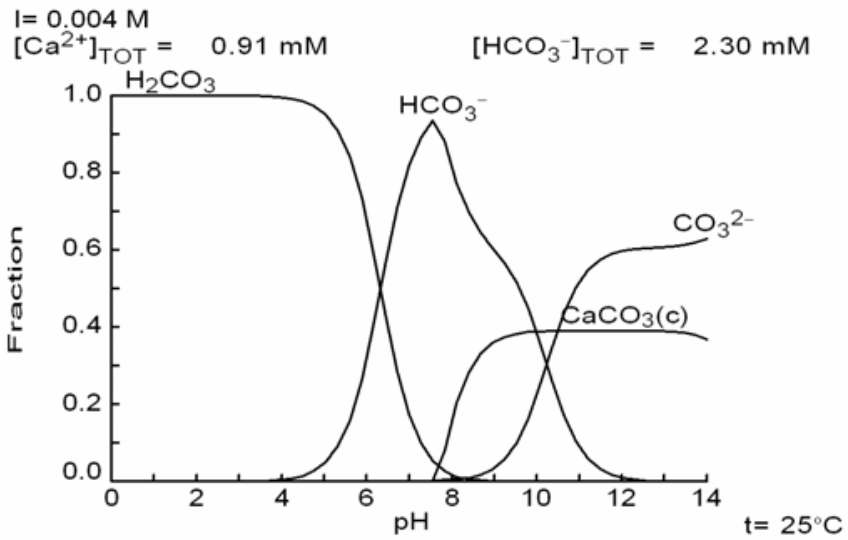

d) En condiciones de equilibrio del Mes 6

Fig. 6: Diagramas de distribución de especies carbonatadas: ácido carbónico, bicarbonatos y carbonatos, en presencia de calcio. 
Se observa que el incremento de la concentración del ión calcio favorece la formación del carbonato de calcio a partir de valores de $\mathrm{pH}$ de 7.7, debajo del $\mathrm{pH}$ equilibrio que es de 8.6, por lo que se deben buscar alternativas distintas al carbonato de calcio e hidróxido de calcio, para ajustar el equilibrio químico del agua (Fig. 6d).

Con base en los valores del $\mathrm{pH}$ de saturación calculados por Mach y el gráfico de Tillmans correspondiente, es posible establecer el equilibrio del agua al agregar un agente inhibidor como el hidróxido de calcio o el carbonato de calcio. Las concentraciones requeridas (tabla 2) para adecuar la calidad química del agua se obtuvieron considerando el ácido carbónico en exceso.

Los datos del último muestreo correspondientes al mes en que finaliza la época de estiaje, indican que el proceso de control de la agresividad más conveniente es por aeración, de esta forma es posible disminuir la concentración del ácido carbónico, sin incrementar la de los iones bicarbonato y calcio, evitando así la formación de carbonato de calcio.

Considerando los resultados calculados en cuanto al pH de saturación, y con excepción del último mes, en el que es conveniente un proceso de aeración, el agua seguiría manteniendo su propiedad potable una vez que se lograra adecuar su química con calhidra o cal.

\section{CONCLUSIONES}

1. El agua potable del tanque posee un carácter corrosivo, lo cual afecta a la infraestructura de almacenamiento y distribución, sin embargo, su calidad fisicoquímica y microbiológica cumple con las Normas Oficiales Mexicanas para su uso y consumo humano.

2. El método de Mojmir Mach resultó ser la mejor opción con respecto al índice de saturación de Langelier y al índice de estabilidad de Ryznar. Mach identifica y cuantifica la tendencia corrosiva del agua, aportando los elementos para establecer el equilibrio químico del agua con base en carbonato de calcio e hidróxido de calcio, o aeración.

3. La tendencia corrosiva por dióxido de carbono del agua obtenida teóricamente fue confirmada a través de difracción de rayos $\mathrm{X}$, microscopia electrónica y microanálisis elemental.

4. Agrlnc_Agua 2.0 ha mostrado ser una herramienta confiable y simple en su manejo, que promueve el uso del método cuantitativo de Mojmir Mach el cual posee un mayor sustento teórico con relación a los métodos cualitativos.

5. Para confirmar la tendencia agresiva del agua, se recomienda utilizar el método de Mach conjuntamente con los índices de saturación y estabilidad. Es conveniente monitorear el agua del tanque de distribución durante un mayor periodo, para establecer las mejores condiciones de equilibrio químico. Adicionalmente, los valores de $\mathrm{CO}_{2}$ en equilibrio y la fuerza iónica pueden constatar la aplicación de aereación para mantener el equilibrio.

6. La presencia de manganeso y otros metales en la red de distribución de cemento reforzado, sugiere evitar periodos largos de interrupción en el abastecimiento de agua.

\section{AGRADECIMIENTOS}

A la Secretaría de Investigación y Estudios Avanzados de la Universidad Autónoma del Estado de México por el apoyo y financiamiento a este proyecto; así como al organismo operador Agua y Saneamiento de Toluca, por las facilidades otorgadas.

\section{REFERENCIAS}

Alfaro, R. y otros cuatro autores; Determinación de la agresividad del agua subterránea en la cuenca de Cuitzeo, Michoacán, Ciencia Nicolaita: 37, 123-131 (2004). 
Allende, A.V.; Apuntes sobre Química del Agua. I.S.P.J.A.E. Habana, Cuba (1976).

APHA-AWWA-WPFC; Standard methods for the examination of water and wastewater. Washington, D.C. (1998).

CCA-Centro Canario del Agua; Índices de Langelier, Ryznar y Puckorius: método de cálculo, notas e interpretación de resultados. (en línea), 2005. http:// www.fcca.es/docs/metodos.htm. Acceso: 28 de octubre (2005).

CNA-Comisión Nacional del Agua; Rehabilitación de pozos. Libro III, 2.1. México. (1994a).

CNA-Comisión Nacional del Agua; Protección catódica y recubrimientos anticorrosivos. Manual de diseño de agua potable, alcantarillado y saneamiento. Libro V, 3.5.1. México. (1994b).

Delion, N.; G. Mauguin y P. Corsin; Importance and impact of post treatments on design and operation of SWRO plants, Desalination: 165, 323-334 (2004).

FPTCDW-Federal-Provincial-Territorial Comitee on Drinking Water; Corrosion control in drinking water distribution Systems. Health Canada, 2007, (en línea) http://www.hc-sc.gc.ca/ewhsemt/ alt formats/hecs-sesc/pdf/pubs/water-eau/consultation/corrosion/corrosion e.pdf. Acceso enero 29 de (2008).

GQ-Gouvernement du Qébec; Guide de conception des installations de production d'eau potable. Volume 1. 2001, modifiés le 4 décembre 2006a, (en línea). http://www.menv.gouv.qc.ca/ eau/potable/guide/index.htm. Acceso: enero 29 de (2008).

GQ-Gouvernement du Québec; Guide de conception des installations de production d'eau potable. Volume 2. 2001, modifiés le 4 décembre 2006b, (en línea). http://www.menv.gouv.qc.ca/ eau/potable/guide/index.htm. Acceso: enero 29 de (2008).

Hamrouni, B. y M. Dhabi; Calco-carbonic equilibrium calculation, Desalination: 152, 167-174 (2002).

Huang, Y.H. y T.C. Zhang; Effects of dissolved oxygen on formation of corrosion products and concomitant oxygen and nitrate reduction in zero-valent iron systems with or without aqueous $\mathrm{Fe}^{2+}$. Water Research: 39, 1751-1760 (2005).

Imran, S.A. y otros cuatro autores; Modified Larsons ratio incorporating Temperature, Water Age, and electroneutrality effects on red water release, J. Environmental Engineering: 131(11), 1514-1520 (2005).

La Motta, E. y S. Chinthakuntla; Corrosion control of drinking water using tray aerators, J. Environmental Engineering: 122(7), 640-648 (1996).

Letterman, R.D., M. Hadad y C.T. Driscoll; Lismestone contactors. Steady-state design relationships, J. Environmental Engineering: 117(3), 339-358(1991).

Masschelein, W.J.; Unit processes in drinking water treatment. Ed. Marcel Dekker, Inc. New York (1982).

NOM-127-SSA1-1994; Modificaciones a la norma mexicana, Salud ambiental. Agua para uso y consumo humano. Limites permisibles de calidad y tratamiento a que se debe someterse el agua para su potabilización, Secretaría de Salud. México (2000).

NRC-National Research Council; Drinking water distribution systems: assessing and reducing risks. Ed. The National Academies. (en linea) 2006. http://www.nap.edu/catalog/11728.html. Acceso: enero 30 de (2008). 
Puigdomenech, I., A. Zagorodni, M. Wang y M. Muhummed;. Program Medusa (Make Equilibrium Diagrams Using Sophisticated Algoritms. Royal Institute of Technology, Inorganic and Materials Chemistry, Sweden, 1999.(en linea). http://www.kemi.kth.se/medusa Acceso: octubre 11 de (2007)

Rahman, S., B.C. McDonald y G.A. Gagnon; Impact of secondary disinfectants on copper corrosion under stagnation conditions, J. Environmental Engineering: 133(2), 180-185 (2007).

Rodier, J.; Análisis de las aguas naturales, aguas residuales, agua de mar, Ediciones Omega S.A. (1998).

Sarin, P., V.L. Snoeyink, D.A. Lytle y W.M. Kriven; Iron Corrosion scales: Model for scale growth, iron release, and colored water formation, J. Environmental Engineering: 130(4), 364-373 (2004).

Shock, M.R.; Internal corrosion and deposition control. Chapter 17 in Water Quality and Treatment, $4^{\text {th }}$ Ed. Edited by American Water Works Association, McGraw-Hill (1990).

Trejo, M.C. y otros cuatro autores; Aseguramiento metrológico de la planta potabilizadora de agua del sistema Cutzamala, Comisión Nacional de Agua. Simposio de Metrología. Querétaro, México, 25 Octubre (2004).

Trujillo, E., V. Martínez y N.S. Flores; Adecuación de la calidad química del agua potable para la infraestructura hidráulica con apoyo informático en Medioambiente en Iberoamérica. Visión desde la Física y la Química en los albores del siglo XXI. Tomo I. Gallardo, J. Editor. Sociedad Iberoamericana de Física y Química Ambiental (2006)

Zhang, Z. J.E. Stout, V.L. Yu y R. Vidic; Effect of pipe corrosion scales on chlorine dioxide consumption in drinking water distribution systems, Water Research: 42, 129-136 (2008). 
